\title{
Phase II Pragmatic Randomized Controlled Trial of Patient-Led Therapies (Mirror Therapy and Lower-Limb Exercises) During Inpatient Stroke Rehabilitation
}

\author{
Sarah Tyson, PhD ${ }^{1,2}$, Jack Wilkinson, MSc ${ }^{1,3}$, Nessa Thomas ${ }^{1,2}$, Ruud Selles, PhD $^{4}$, \\ Candy McCabe, PhD ${ }^{5}$, Pippa Tyrrell, MD ${ }^{1,3}$, and Andy Vail, MSc ${ }^{1,3}$
}

\begin{abstract}
Background and objective. Patient-led therapy has the potential to increase the amount of therapy patients undertake during stroke rehabilitation and to enhance recovery. Our objective was to assess the feasibility and acceptability of 2 patient-led therapies during the acute stages of stroke care: mirror therapy for the upper limb and lower-limb exercises for the lower limb. Methods. This was a blind assessed, multicenter, pragmatic randomized controlled trial of patient-led upper-limb mirror therapy and patient-led lower leg exercises. Stroke survivors with upper and lower limb limitations, undergoing inpatient rehabilitation and able to consent were recruited at least I week poststroke. Results. Both interventions proved feasible, with $>90 \%$ retention. No serious adverse events were reported. Both groups did less therapy than recommended; typically 5 to 15 minutes for 7 days or less. Participants receiving mirror therapy $(n=63)$ tended to do less practice than those doing lower-limb exercises $(n=31)$. Those with neglect did $69 \%$ less mirror therapy than those without $(P=.02)$, which was not observed in the exercise group. Observed between-group differences were modest but neglect, upper-limb strength, and dexterity showed some improvement in the mirror therapy group. No changes were seen in the lower-limb group. Conclusions. Both patient-led mirror therapy and lower-limb exercises during inpatient stroke care are safe, feasible, and acceptable and warrant further investigation. Practice for 5 to 15 minutes for 7 days is a realistic prescription unless strategies to enhance adherence are included.
\end{abstract}

\section{Keywords}

stroke, patient-led, mirror therapy, exercise

\section{Introduction}

It is well established that the amount of therapy received is a key factor in recovery from stroke; the more the better, and the most-effective physical interventions are exercise and the intensive practice of functional tasks. ${ }^{1,2}$ Despite this, most stroke patients spend most of their day during inpatient stroke care inactive and alone. ${ }^{3-5}$ They receive low levels of therapy, ${ }^{4,6}$ which are insufficient to provide the intensity of practice needed to develop motor skills. ${ }^{1}$ There is, therefore, an imperative to develop interventions that enable patients undertake more exercise and practice. As a major increase in staffing levels is unlikely, this needs to be achieved without increasing the demands on staff. One possibility is patient-led therapy (also known as independent practice or self-directed therapy) in which the patients undertake tasks or exercises prescribed by a health care professional outside the traditional one-to-one treatment sessions with a therapist. This type of therapy is effective ${ }^{7-9}$ and widely used in community-based rehabilitation but is rarely attempted during inpatient rehabilitation., ${ }^{4,10}$

We undertook a pragmatic phase II feasibility trial of 2 types of patient-led therapy during inpatient stroke care to gather information to inform a possible phase III trial. Specifically, we assessed completion rates, adverse events, and patient adherence and gathered preliminary data concerning any impact on impairment and activity. The 2

\footnotetext{
'University of Manchester, Manchester, UK

${ }^{2}$ School of Nursing Midwifery \& Social Word, Manchester, UK

${ }^{3}$ Salford Royal NHS Foundation Trust, UK

${ }^{4}$ Erasmus MC, Rotterdam, the Netherlands

${ }^{5}$ University of the West England, Bristol and Royal National Hospital for Rheumatic Diseases NHS Foundation Trust, Bath, UK

\section{Corresponding Author:}

Sarah Tyson, PhD, Stroke Research Centre, University of Manchester, Jean McFarlane Building, Oxford Rd, Manchester MI3 9PL, UK. Email: Sarah.tyson@manchester.ac.uk
} 


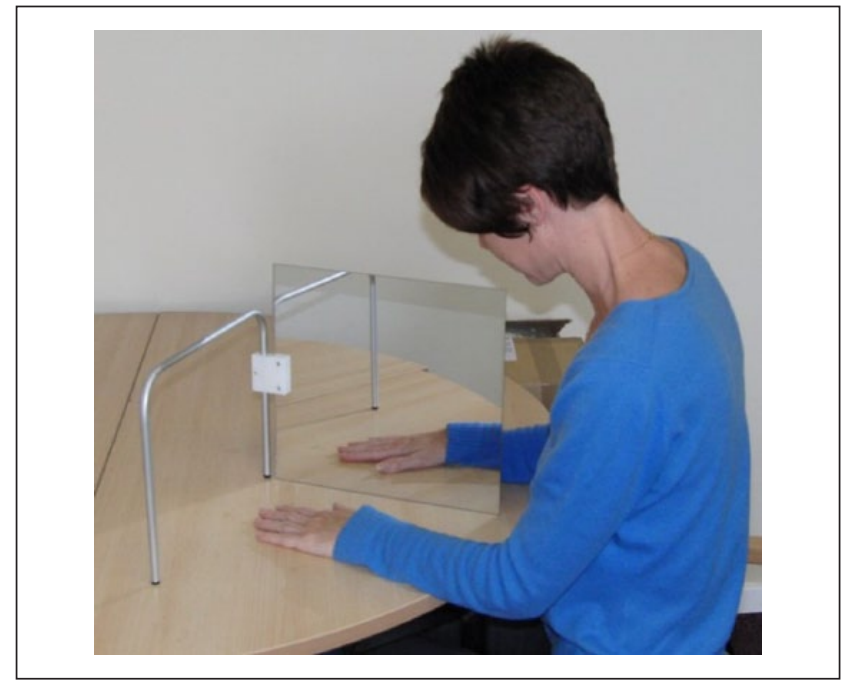

Figure I. The mirror in use.

interventions tested were mirror therapy for the upper limb and exercises for the lower limb.

Mirror therapy shows promise as a treatment to improve upper-limb function after stroke. ${ }^{11} \mathrm{~A}$ recent Cochrane Review of mirror therapy for people with stroke ${ }^{11}$ involving 14 studies and 567 participants concluded that it can improve upper-limb motor function, activities of daily living, and when tested, neglect and pain as an adjunct to normal rehabilitation. However, most participants were in the chronic stages of stroke, and there are limited data on the value of mirror therapy soon after stroke. Furthermore, details of how the mirror therapy was delivered are often lacking. Mirror therapy involves the seated patient placing their sound arm in front of a table-mounted mirror with their weak hand behind it. When the patient looks in the mirror, the reflected image of their sound arm moving gives the visual illusion they are watching their weak hand move. This illusion is thought to enable the patient to move their weak limb more easily, although the mechanism is unclear. A recent trial has demonstrated that patient-led mirror therapy is feasible in a community setting when undertaken by people with chronic strokes. ${ }^{12}$

Patient-led exercise is another possible way to increase patients' activity levels. Exercise can improve both impairments and activity/disability levels poststroke ${ }^{2,13}$ but most trials involve supervised, community-based exercise involving people with subacute or chronic stroke.

\section{Methods}

\section{Design}

A pragmatic assessor-blinded multicenter controlled trial with stratified imbalanced randomization (allocation ratio 2:1) was approved by the National Ethics Service and
University Committees. Participants were recruited through the North-West Stroke Local Research Network from inpatient stroke rehabilitation services in 12 hospitals across North-West England. Eligible participants had experienced a stroke at least 1 week previously, with no premorbid conditions limiting upper- or lower-limb function; had sufficient cognitive and communication skills to give informed consent; and had upper- and lower-limb weakness that limited activity.

As there were no previously reported data for patient-led therapy during acute stroke, a formal power calculation was not appropriate. Instead, we recruited sufficient numbers to enable a sufficiently precise estimate of the variability (standard deviation) of study end points for future sample size calculations and sufficient replications of the protocol to assess feasibility. At least 55 participants recruited to the mirror therapy would enable these objectives, including a $10 \%$ dropout. To allow initial evaluation of effect, we also included a control group using a 2:1 allocation ratio (mirror:control).

Following informed consent, participants undertook baseline assessments before randomization using an independent Web-based randomization service, so that allocation was concealed. The strata for randomization were defined by upper-limb weakness (motricity index $<48$ or $48+)$ and the presence of neglect $(<44$ on the Star Cancellation test). Random block sizes preserved allocation concealment. A blinded assessor repeated the assessments at the end of the treatment period (4 weeks) and at followup (4 weeks after the end of treatment).

\section{Interventions}

All participants received usual care and were randomized to also receive either patient-led mirror therapy or patient-led lower-limb exercise (without a mirror). This ensured that both groups received similar attention from the trial and clinical teams, thereby balancing placebo effects. Because the effects of exercise are specific to the areas exercised, exercising the lower limb would not affect the upper limb, and vice versa, so the lower-limb data from the mirror therapy group acted as the control for the group receiving lower-limb exercises, and the upper-limb data from the exercise group acted as the control for the mirror therapy group.

The mirror therapy intervention was based on that used by Michielsen et $\mathrm{al}^{12}$ but because the interventions needed to be "fit-for-purpose" for acute care settings, the trial team worked with consultation groups of stroke survivors and stroke therapists throughout the trial to ensure that the treatment and trial protocols were feasible and acceptable for the patients and to fit within every-day clinical practice (Figures 1 and 2). It is well established that exercise needs to be feasible yet challenging to improve strength and motor 


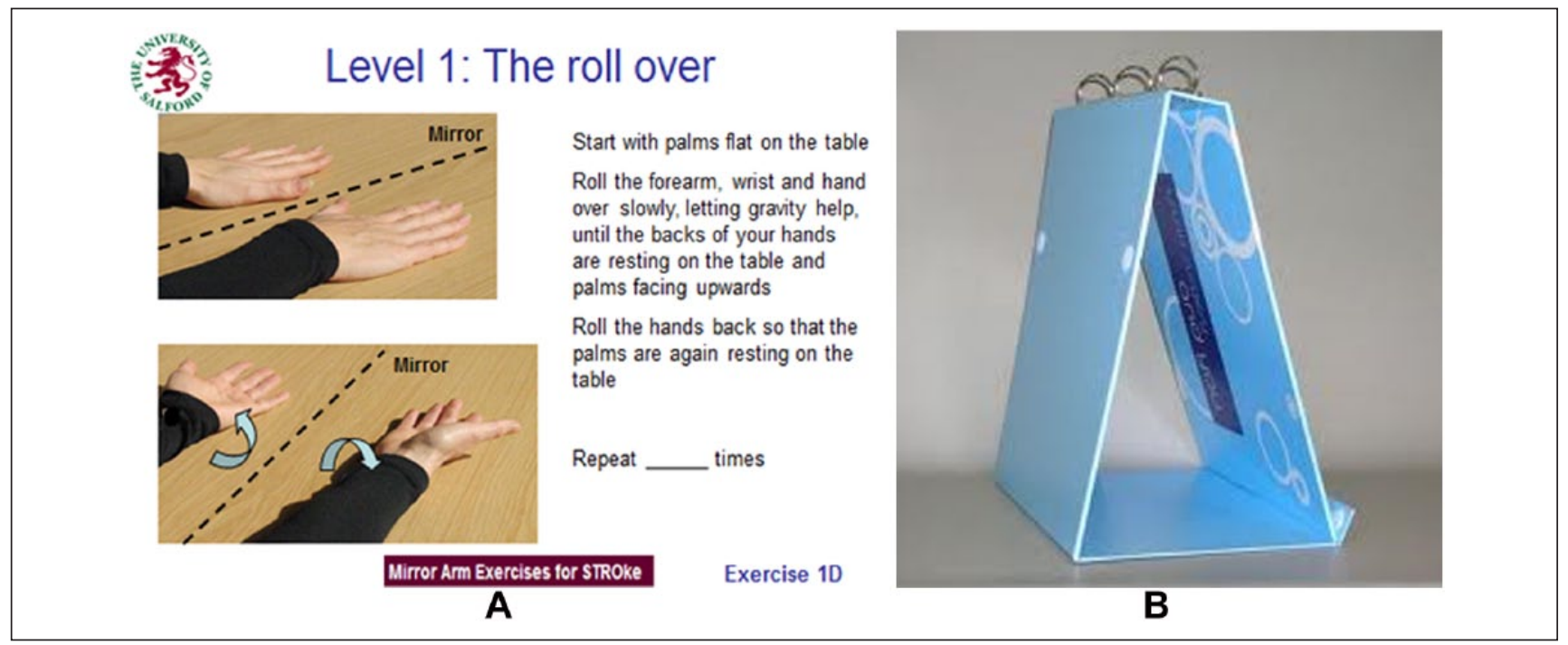

Figure 2. The exercise booklets: $(A)$ example of the exercise sheets and $(B)$ the free-standing folder.

control, so the therapies were individualized to each patient to accommodate the wide range of abilities. For each intervention, there were 4 levels of exercises. For the mirror therapy, fingers, wrist, and elbow movements; reaching; and at the highest level, functional activities were exercised. The lower-limb exercises involved the ankles, knees, and hips.

- Level 1 involved flexion, extension, abduction, and adduction movements with the limb fully supported.

- Level 2 involved multiplanar multiple joint movements, some against gravity.

- Level 3 involved movements against gravity.

- Level 4 involved functional activities such as grasping and moving objects (for the upper limb) and standing up and sitting down (for the lower limb).

The most appropriate exercises were selected for each patient by the treating clinician following their assessment, and instructions (with aphasia-friendly photos and written instruction) on how to perform them were put in a freestanding ring binder folder, which the patient kept to act as an aide-memoire (Figure 2). As the patient progressed, the redundant exercises were removed and new ones were added by the treating clinician.

For each participant a local treating clinician was identified to take day-to-day responsibility for supervising the interventions. The trial therapists initially taught each participant and local clinician how to do the interventions through instruction and demonstrations and selected the initial exercises with them. Participants were asked to undertake up to 30 minutes of daily practice. It was unlikely that 30 minutes of concentrated practice would be tolerable, and so ways in which the practice could be undertaken "a little and often" and fitted into the routine of the rehabilitation unit (eg, identifying times in the day/timetable for the patient to practice) were negotiated. The local clinician checked on and encouraged the patient's practice, dealt with any problems, and progressed the exercises as necessary. They also encouraged participants to complete the practice log sheet and completed a log of their own input. Patients who were discharged before the end of the 4-week treatment were encouraged to continue with their allocated intervention. The trial therapist rang or visited the patient twice a week to monitor adherence and the patient's progression on to more advanced exercises, as necessary. Wherever possible, a carer or relative was also taught about the treatment and encouraged to assist the patient as necessary and able.

\section{Outcome Measures}

Assessments were made at baseline, at the end of treatment (4 weeks after baseline), and at follow up ( 8 weeks after baseline). We measured the upper limb to assess the mirror therapy and the lower limb to assess the lower-limb exercises, as follows:

- Upper and lower-limb weakness (Motricity Index ${ }^{14}$ and grip strength ${ }^{15}$ )

- Upper and lower-limb sensation (Rivermead Assessment of Sensory Perception ${ }^{16}$ )

- Spasticity of biceps and gastrocnemius for the upperand lower-limb, respectively (Modified Ashworth Scale ${ }^{17}$ )

- Neglect (Star Cancellation test ${ }^{18}$ )

Specifically for the upper limb, we measured dexterity (Box and Block ${ }^{19}$ ) and activity (Action Research Arm Test $^{19}$ ). For lower limbs, we also measured mobility 
(Rivermead Mobility Index ${ }^{20}$ ) and balance (Brunel Balance Assessment ${ }^{21}$ ). We monitored participants' adherence using a daily self-reported exercise log. Adverse events were monitored during the treatment phase and reported using standardized forms. We also intended to record activity in everyday life (Motor Activity Log), but human error led to uninterpretable data.

\section{Analysis}

In addition to descriptive analyses, we undertook exploratory comparisons of outcome using $t$ tests and 95\% confidence intervals (CIs) for the difference in mean change from baseline to 4 and 8 weeks. To explore whether potential responders could be identified, plausible exploratory subgroup analyses of neglect, sensory impairment, weakness, and balance at each time point were undertaken. Patients were excluded from a given analysis if their corresponding measurement was missing; in other respects, we used an intention-to-treat analysis. Multiple linear regression identified factors influencing treatment adherence. Treatment time was log transformed to satisfy the assumptions of the regression model. The included predictors were age, side of weakness, time since stroke, treatment group, neglect, and sensation.

\section{Results}

A total of 94 participants were recruited: 63 and 31 to the mirror therapy and lower-limb exercises, respectively. Retention rate was high $(90 \%)$, which can be seen in Figure 3. Demographic and clinical characteristics and baseline assessments are shown in Tables 1 and 2 . The cohort was representative of patients in inpatient stroke care with a wide range of abilities and no major differences between groups. No serious adverse events were reported. There were 8 reports of short-lived upper- and lower-limb aches or limb tightness.

A total of 1801 treatment sessions were recorded; 82 participants $(87 \%)$ recorded at least 1 session-51 $(81 \%)$ in the mirror therapy and $30(97 \%)$ in the lower-limb exercises group. It is not known whether participants who did not complete the exercise $\log$ s did not do any exercise or just did not complete the log. The mean (SD) number of days when the patient-led therapies were performed was 14 $(\mathrm{SD}=10$; range $=1-41)$ days $-12.9(\mathrm{SD}=9.3)$ days for the mirror group and 15.2 (SD = 9.5) days for the lower-limb group - but this difference was not significant $(P=.294$; $95 \% \mathrm{CI}=-6.64,2.04)$. In all, $21(26 \%)$ patients continued to exercise and record beyond the designated 28-day period, but most practiced for 7 days or fewer $(n=29,35 \%)$. This was slightly higher in the mirror therapy group $(n=20$, $39 \%)$ than in the lower-limb exercise group $(n=10,30 \%)$. Only $2(1 \%)$ mirror therapy participants exercised for the recommended 28 days, whereas 5 (15\%) lower-limb exercise participants achieved this. The most common duration of exercise was 5 to 15 minutes $(\mathrm{n}=1054,59 \%)$ per session. Only $1 \%(n=21)$ of mirror therapy sessions and $3 \%$ $(n=40)$ of lower-limb exercise sessions lasted for the recommended 30 minutes.

Linear regression suggested that participants with neglect in the mirror therapy group practiced less than those without neglect. After adjusting for the other factors, mirror therapy group participants with neglect achieved only $31 \%$ $(P=.02 ; 95 \% \mathrm{CI}=12 \%, 82 \%)$ of the practice time of those without neglect. There was an interaction between treatment group and neglect $(P=.052)$, indicating that this disadvantage was not present in the lower-limb exercise group.

Outcome assessments at 4 and 8 weeks are shown in Tables 2, 3, and 4 and as standardized mean differences in Figure 4. Neglect, upper-limb weakness, and dexterity showed some (nonsignificant) improvement for the mirror therapy group compared with the lower-limb group at the end of treatment, which was sustained at follow-up. The mirror therapy group showed less improvement in upper-limb sensation and activity than the control group. All between-group differences were small relative to the variability of the end points. There was no evidence of differences in the lowerlimb outcome measures (Table 4, Figure 4).

The exploratory subgroup analyses suggested that people with neglect who received mirror therapy had less neglect at follow-up than those who received the lowerlimb exercises. The mean change (in Star Cancellation test) was $23.5(\mathrm{SD}=11.3)$ and $10.0(\mathrm{SD}=8.0)$ stars, respectively, and the difference in means was 13.5 stars $(P=.03$; $95 \%$ CI $=1.7,25.2)$. All other explorations were nonsignificant.

\section{Discussion}

This study shows that patient-led mirror therapy and lowerlimb exercises are safe, feasible, and acceptable to patients during inpatient stroke care. Few participants achieved the recommended 30 minutes of daily practice for 28 days, however. Most practiced for 5 to 15 minutes for 7 days out of the 28. The presence of neglect had an impact on the amount of patient-led mirror therapy undertaken. Differences between groups were small, but neglect, upperlimb strength, and dexterity showed some greater improvement in the mirror therapy group than in the controls, whereas sensation and upper-limb activity improved less than in the lower-limb group.

As an unpowered trial, significant results would not be expected. However, our sample size was larger than that of most previous trials, some of which have reported positive results. ${ }^{4,7,8}$ The current trial differs from previous reports in several other methodological details. This is the first trial to specifically involve people in the acute stages of stroke. A 


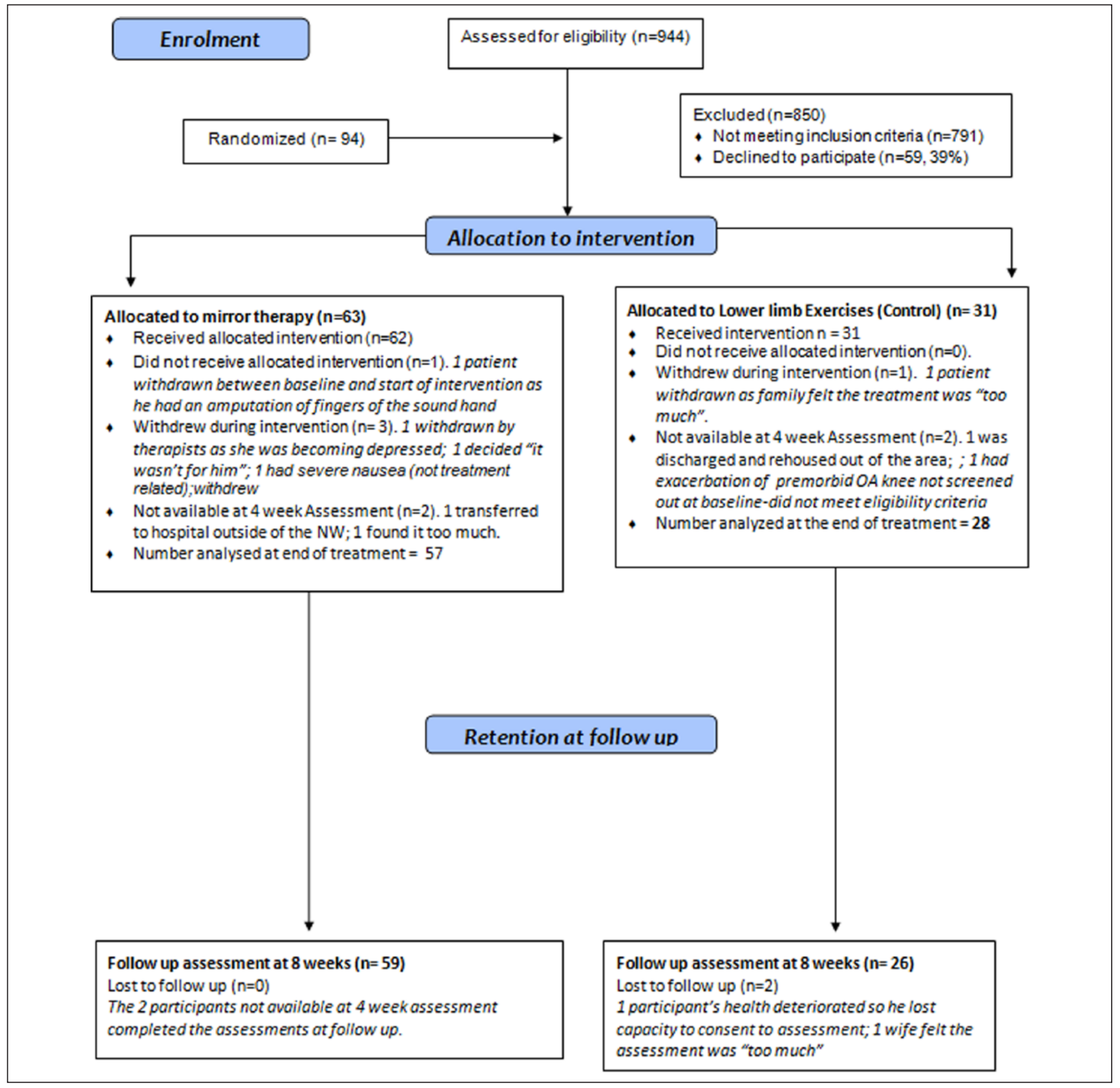

Figure 3. Consort flow diagram.

Table I. Baseline Demographic and Clinical Characteristics.

\begin{tabular}{|c|c|c|c|c|}
\hline Characteristic & & Total Group (n = 94) & Mirror Therapy $(n=62)$ & Lower-Limb Exercises $(n=31)$ \\
\hline \multirow[t]{2}{*}{ Age (years) } & Median (range) & $66(26-92)$ & $66(26-88)$ & $65(40-91)$ \\
\hline & Mean (SD) & $64(14)$ & $64(15)$ & $64(13)$ \\
\hline Sex & Male & $60(65 \%)$ & $37(60 \%)$ & $23(74 \%)$ \\
\hline \multirow[t]{2}{*}{ Time since stroke (days) } & Median (range) & $19(7-133)$ & $18(7-76)$ & $29(7-133)$ \\
\hline & Mean (SD) & $28.8(22)$ & $26(18)$ & $35(27)$ \\
\hline Stroke type & Ischemic & 76 (82\%) & $50(81 \%)$ & $26(84 \%)$ \\
\hline Side of weakness & Dominant side & 35 (38\%) & $25(40 \%)$ & $10(32 \%)$ \\
\hline Side of weakness & Right side & 38 (4I\%) & 27 (44\%) & II (35\%) \\
\hline
\end{tabular}

Abbreviation: SD, standard deviation. 
Table 2. Baseline Assessments of the Recruited Participants.

\begin{tabular}{|c|c|c|c|c|}
\hline \multicolumn{2}{|l|}{ Assessment } & \multirow{2}{*}{ Total Group $(\mathrm{n}=94)$} & \multirow{2}{*}{ Mirror Therapy $(n=62)$} & \multirow{2}{*}{ Lower-Leg Exercises $(n=3 I)$} \\
\hline \multirow{3}{*}{$\begin{array}{l}\text { Upper limb } \\
\text { Neglect (Star Cancellation) }\end{array}$} & & & & \\
\hline & Median (range) & & $55(9-56)$ & $54(8-56)$ \\
\hline & Mean (SD) & $47(14)$ & $47(15)$ & $48(12)$ \\
\hline \multirow[t]{2}{*}{ Weakness (Motricity Index) } & Median (range) & $43(1-93)$ & $44(1-93)$ & $43(1-85)$ \\
\hline & Mean (SD) & $40(30)$ & $40(32)$ & $39(29)$ \\
\hline \multirow[t]{2}{*}{ Weakness (grip strength) } & Median (range) & $0(0-20)$ & $0(0-20)$ & $0(0-12)$ \\
\hline & Mean (SD) & $3(6)$ & $4(7)$ & $2(3)$ \\
\hline \multirow{2}{*}{$\begin{array}{l}\text { Sensation (Rivermead Assessment } \\
\text { of Sensory Perception) }\end{array}$} & Median (range) & $19(0-24)$ & $22(0-24)$ & $16(0-24)$ \\
\hline & Mean (SD) & $16(9)$ & $17(9)$ & $14(8)$ \\
\hline \multirow{2}{*}{$\begin{array}{l}\text { Spasticity (Modified Ashworth } \\
\text { Scale of biceps) }\end{array}$} & Median (IQR) & $0(0-2)$ & $0(0-I)$ & $0(0-2)$ \\
\hline & Mean (SD) & I (I) & I (I) & I (I) \\
\hline \multirow[t]{2}{*}{ Dexterity (Box and Block) } & Median (range) & $0(0-36)$ & $0(0-36)$ & $0(0-29)$ \\
\hline & Mean (SD) & $5(9)$ & $5(9)$ & $4(9)$ \\
\hline \multirow{2}{*}{$\begin{array}{l}\text { Actvity (Action Research Arm } \\
\text { Test) }\end{array}$} & Median (range) & $0(0-57)$ & $0(0-57)$ & $0(0-48)$ \\
\hline & Mean (SD) & $12(17)$ & $13(18)$ & $10(15)$ \\
\hline \multicolumn{5}{|l|}{ Lower limb } \\
\hline \multirow[t]{2}{*}{ Weakness (Motricity Index) } & Median (range) & $54(1-92)$ & $59(1-92)$ & $48(1-92)$ \\
\hline & Mean (SD) & $50(27)$ & $52(27)$ & $46(28)$ \\
\hline \multirow{2}{*}{$\begin{array}{l}\text { Sensation (Rivermead Assessment } \\
\text { of Sensory Perception) }\end{array}$} & Median (range) & $19(0-24)$ & $20(0-24)$ & $19(0-24)$ \\
\hline & Mean (SD) & $16(8)$ & $17(8)$ & $15(9)$ \\
\hline \multirow{2}{*}{$\begin{array}{l}\text { Spasticity (Modified Ashworth } \\
\text { Scale of tibialis anterior) }\end{array}$} & Median (IQR) & $0(0-2)$ & $0(0-I)$ & $0(0-2)$ \\
\hline & Mean (SD) & I (I) & I (I) & I (I) \\
\hline \multirow{2}{*}{$\begin{array}{l}\text { Balance (Brunel Balance } \\
\text { Assessment) }\end{array}$} & Median (IQR) & $4(0-12)$ & $4(3-5)$ & $3(1-4)$ \\
\hline & Mean (SD) & $4(3)$ & $4(3)$ & $4(3)$ \\
\hline \multirow{2}{*}{$\begin{array}{l}\text { Mobility (Rivermead Mobility } \\
\text { Index) }\end{array}$} & Median (range) & I (0-|4) & I (0-14) & I (0-7) \\
\hline & Mean (SD) & $3(3)$ & $3(3)$ & $2(3)$ \\
\hline
\end{tabular}

Abbreviations: SD, standard deviation; IQR, interquartile range.

Table 3. Change in Upper-Limb Outcomes at the End of the Intervention and Follow-up.

\begin{tabular}{|c|c|c|c|c|}
\hline Measure & $\begin{array}{l}\text { Mirror Therapy, } \\
\text { Mean (SD) }\end{array}$ & $\begin{array}{l}\text { Lower-Limb Exercises, } \\
\text { Mean (SD) }\end{array}$ & Difference $(95 \% \mathrm{Cl})$ & $P$ Value \\
\hline \multicolumn{5}{|l|}{ At the end of the intervention ( 4 weeks) } \\
\hline Neglect (Star Cancellation) & $4.4(10.8)$ & $2(7.4)$ & $2.4(-2.1,6.8)$ & .30 \\
\hline Weakness (Motricity Index) & $9.1(18.3)$ & $6.8(16.8)$ & $2.4(-5.8,10.6)$ & .57 \\
\hline Weakness (grip strength) & $1.7(4.9)$ & $2.8(5.7)$ & $-1.0(-3.4,1.4)$ & .41 \\
\hline $\begin{array}{l}\text { Sensation (Rivermead Assessment of Sensory } \\
\text { Perception) }\end{array}$ & $0.3(7.7)$ & $2.4(6.2)$ & $-2.1(-5.4,1.2)$ & .21 \\
\hline Spasticity (Modified Ashworth Scale of biceps) & $-0.0(1.0)$ & $-0.3(1.1)$ & $0.2(-0.3,0.7)$ & .36 \\
\hline Dexterity (Box and Block test) & $8.2(12.7)$ & $6.0(11.1)$ & $2.2(-3.3,7.8)$ & .42 \\
\hline Activity (Action Research Arm test) & $6.9(13.9)$ & $8.3(12.1)$ & $-1.4(-7.6,4.7)$ & .64 \\
\hline \multicolumn{5}{|l|}{ At the end of follow-up ( 8 weeks) } \\
\hline Neglect (Star Cancellation) & $5.9(11.3)$ & $3.2(5.4)$ & $2.7(-1.9,7.4)$ & .24 \\
\hline Weakness (Motricity Index) & $12.9(20.8)$ & $10.5(19.9)$ & $2.3(-7.2,12.0)$ & .63 \\
\hline Weakness (grip strength) & $2.3(6.8)$ & $3.6(5.4)$ & $-1.3(-4.4,1.7)$ & .40 \\
\hline $\begin{array}{l}\text { Sensation (Rivermead Assessment of Sensory } \\
\text { Perception) }\end{array}$ & $2.4(6.5)$ & $3.3(7.4)$ & $-0.9(-4.1,2.3)$ & .56 \\
\hline Spasticity (Modified Ashworth Scale of biceps) & $-0.1(1.1)$ & $0.1(1.6)$ & $-0.2(-0.8,0.4)$ & .51 \\
\hline Dexterity (Box and Block test) & $9.3(14.9)$ & $7.0(12.3)$ & $2.4(-4.3,9.0)$ & .48 \\
\hline Activity (Action Research Arm test) & $8.5(14.5)$ & $8.8(13.8)$ & $-0.3(-7.0,6.4)$ & .93 \\
\hline
\end{tabular}

Abbreviations: SD, standard deviation; $\mathrm{Cl}$, confidence interval. 

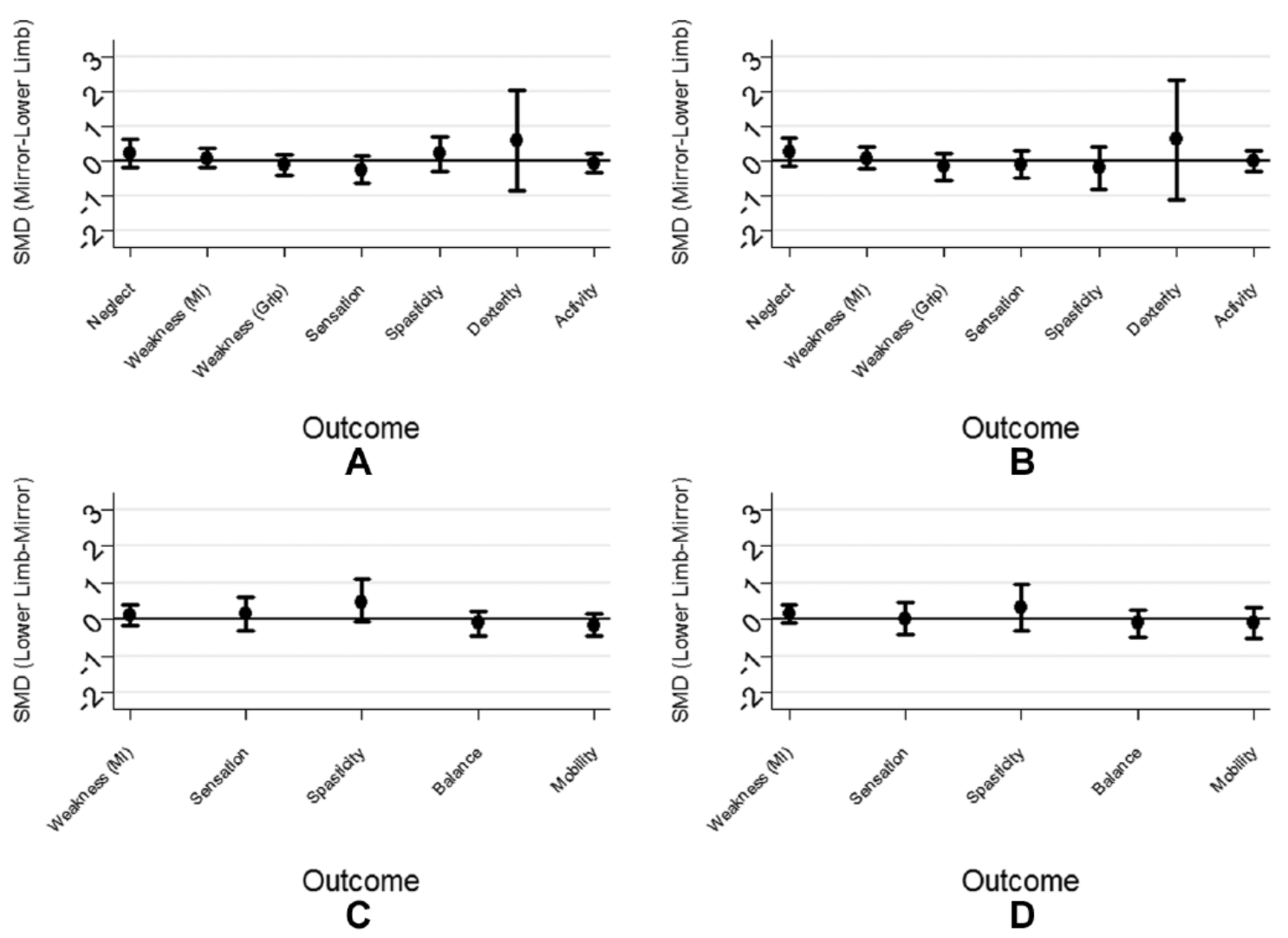

Figure 4. Standardized mean differences (SMD) for upper-limb outcomes (mirror therapy minus control) at (A) 4 weeks and (B) 8 weeks. Lower-limb outcomes (lower-limb exercises minus mirror therapy) at (C) 4 weeks and (D) 8 weeks. The error bars indicate the $95 \%$ confidence intervals.

Abbreviations: MI, Motricity Index.

Table 4. Change in Lower-Limb Outcomes at the End of the Intervention and Follow-up.

\begin{tabular}{|c|c|c|c|c|}
\hline Measure & $\begin{array}{l}\text { Lower-Limb Exercises } \\
\qquad(\mathrm{n}=29)\end{array}$ & Control $(n=57)$ & Difference $(95 \% \mathrm{Cl})$ & $P$ Value \\
\hline \multicolumn{5}{|l|}{ At the end of the intervention ( 4 weeks) } \\
\hline Weakness (Motricity Index) & $13.5(15.4)$ & $10.5(16.4)$ & $3.0(-4.5,10.4)$ & .43 \\
\hline $\begin{array}{l}\text { Spasticity (Modified Ashworth Scale of } \\
\text { gastrocnemius) }\end{array}$ & $0.1(2.0)$ & $-0.5(1.6)$ & $0.6(-0.1,1.4)$ & .11 \\
\hline $\begin{array}{l}\text { Sensation (Rivermead Assessment of Sensory } \\
\text { Perception) }\end{array}$ & $1.7(9.1)$ & $0.4(8.0)$ & $1.3(-2.5,5.1)$ & .51 \\
\hline Mobility (Rivermead Mobility Index) & $2.4(2.8)$ & $3.1(2.9)$ & $-0.7(-2.0,0.7)$ & .32 \\
\hline Balance (Brunel Balance Assessment) & $1.9(1.9)$ & $2.4(3.1)$ & $-0.4(-1.7,0.8)$ & .48 \\
\hline \multicolumn{5}{|l|}{ At the end of follow-up ( 8 weeks) } \\
\hline Motricity Index (Motricity Index) & $20.3(12.5)$ & $16.6(15.0)$ & $3.7(-3.1,10.4)$ & .28 \\
\hline $\begin{array}{l}\text { Sensation (Rivermead Assessment of Sensory } \\
\text { Perception) }\end{array}$ & $1.9(10.6)$ & 1.7 (6.7) & $0.2(-3.6,4.0)$ & .93 \\
\hline $\begin{array}{l}\text { Spasticity (Modified Ashworth Scale of } \\
\text { gastrocnemius) }\end{array}$ & $0.1(1.8)$ & $-0.3(1.7)$ & $0.4(-0.4,1.2)$ & .29 \\
\hline Mobility (Rivermead Mobility Index) & $3.0(2.7)$ & $3.5(3.0)$ & $-0.4(-1.8,0.9)$ & .52 \\
\hline Balance (Brunel Balance Assessment) & $3.7(4.1)$ & $4.1(3.7)$ & $-0.4(-2.2,1.4)$ & .65 \\
\hline
\end{tabular}

Abbreviation: $\mathrm{Cl}$, confidence interval. 
definitive study in this population may require a large sample size because any "signal" from the patient-led therapy may be subsumed by the "noise" of spontaneous recovery and standard care. Using the data from this trial, we calculate that a sample size of 250 patients would be required to detect clinically meaningful differences in a phase III trial.

A methodological difference with previous studies of mirror therapy is that most appear to involve direct supervision from trial therapists. ${ }^{11}$ We used patient-led mirror therapy with light-touch supervision from the clinical therapy team. This provides a pragmatic indication of the potential impact of mirror therapy in everyday clinical life, but it meant that we had little control over clinicians' fidelity to the treatment protocol. There is a growing recognition that these are neglected, but crucial, elements of complex intervention trials. ${ }^{22}$ Clear strategies to ensure fidelity is maintained and monitored in future trials are needed.

Participants' adherence to the interventions was less than expected but perhaps not surprising because it was largely up to the patient when to practice. In the process evaluation accompanying this trial, some participants described how adherence tailed off as they became bored or demotivated by lack of obvious improvement. Others, particularly those with more severe strokes, struggled to complete the therapy without additional help. Thus, an insufficient dose of therapy may be a factor. Adherence has rarely been reported in detail in therapy trials and is generally assumed to be high, given that most trials use directly supervised therapy. Future trials, particularly of the patient-led therapy, should comment on adherence and include strategies to maximize adherence. Our therapy delivery was based on that of Michielsen et al, ${ }^{12}$ who used patient-led mirror therapy for people with chronic stroke at home, with telephone support and weekly face-to-face contact from trial therapists. They had excellent (self-reported) adherence. It may be that participants found mirror therapy easier to undertake at home than in the cramped and busy environment of acute stroke care, particularly while they are still fatigued and overwhelmed by the emotional shock of the stroke. This point was highlighted by some of our participants who continued the mirror therapy after discharge from hospital. Additionally, a few of our participants raised concerns that patient-led therapy may interfere with mainstream rehabilitation. As with the trials of patient-led exercise, participants in the study by Michielsen et $\mathrm{a}^{12}$ had completed their rehabilitation, so participation was a welcome opportunity to access further therapy. This could have increased motivation and adherence. ${ }^{7-9}$

The choice of control is another methodological difference from previous trials of patient-led therapy. Most trials of mirror therapy chose other upper-limb therapies (most commonly exercises without a mirror or a sham mirror) as the control. This provides information about whether the mirror provides an additional benefit to exercise alone but does not indicate the potential advantage of adding mirror therapy to standard clinical care. Because this was our primary interest, we chose an attentional control that did not involve the upper limb. As evaluation of mirror therapy moves from efficacy to clinical effectiveness, clinically relevant control interventions are needed.

Surprisingly, although participants with neglect undertook less mirror therapy than others, there was still a positive effect. Two recent trials have also reported a positive impact of mirror therapy on neglect (as secondary outcomes). ${ }^{23,24}$ This raises an interesting question about the potential mechanism of mirror therapy. Trials of intensive upper-limb exercises with mirror therapy are based on motor learning theory in which intensive practice of functional tasks is key to restoring movement and function. ${ }^{2}$ It is hypothesized that the mirror therapy promotes the neuroplasticity that underlies this process, possibly through visually guided motor imagery and the mirror neuron system. A recent systematic review of imaging studies of the effects of mirror therapy on the brain ${ }^{25}$ partially supports this hypothesis because it indicates that mirror therapy facilitates motor activity contralateral to the affected hand. The mirror neuron system may have a role, but this is not central because only isolated parts of the mirror neuron system are active during mirror therapy. Much more active are areas related to attention and action monitoring, and this may be the mechanism that enhances visuospatial attention in stroke survivors with neglect. However, because nearly all the selected evidence arose from studies of healthy individuals, such hypotheses are speculative. Further research is needed to establish whether the same mechanisms are at work in clinical populations, so the most appropriate patients can be targeted and treatment protocols developed.

\section{Conclusion}

Patient-led mirror therapy and lower-limb exercises are safe, feasible, and acceptable during inpatient stroke rehabilitation and warrant further investigation. Practice for 5 to 15 minutes for 7 days is a realistic prescription unless strategies to enhance adherence are included.

\section{Authors' Note}

Clinical Trial Registration Information: http://www.controlledtrials.com. Unique identifier: ISRCTN29533052.

\section{Declaration of Conflicting Interests}

The authors declared no potential conflicts of interest with respect to the research, authorship, and/or publication of this article.

\section{Funding}

The authors disclosed receipt of the following financial support for the research, authorship, and/or publication of this article: This 
article presents independent research funded by the National Institute for Health Research under its Research for Patient Benefit (RfPB) Programme (Grant Reference Number PB-PG-120818077). The views expressed are those of the author(s) and not necessarily those of the NHS, the NIHR, or the Department of Health.

\section{References}

1. Kwakkel G, van Peppen R, Wagenaar RC, et al. Effects of augmented exercise therapy time after stroke: a meta-analysis. Stroke. 2004;35:2529-2539.

2. Van Peppen RP, Kwakkel G, Wood-Dauphinee S, et al. The impact of physical therapy on functional outcomes after stroke: what's the evidence? Clin Rehabil. 2004;18:833-862.

3. Esmonde T, McGinley J, Wittwer J, et al. Stroke rehabilitation: patient activity during non-therapy time. Aust $J$ Physiother. 1997;43:43-51.

4. Bernhardt J, Chan J, James NI, et al. Little therapy, little physical activity: rehabilitation within the first 14 days of organized stroke unit care. J Rehabil Med. 2007;39:43-48.

5. Bernhardt J, Dewey HM, Thrift AG, et al. Inactive and alone: physical activity in the first 14 days of acute stroke unit care. Stroke. 2004;35:1005-1009.

6. Intercollegiate Stroke Working Party, Royal College of Physicians. National sentinel stroke audit 2010 round 7. https://www.rcplondon.ac.uk/sites/default/files/national sen tinel_stroke_clinical_audit_2010_-_generic_report_0.pdf. Accessed December 19, 2014.

7. Harris JE, Eng JJ, Miller WC, et al. A self-administered Graded Repetitive Arm Supplementary Program (GRASP) improves arm function during inpatient stroke rehabilitation. A multi-site randomized controlled trial. Stroke. 2009;40:2123-2128.

8. Langhammer B, Stanghelle JK, Lindmark B. An evaluation of two different exercise regimes during the first year following stroke: a randomised controlled trial. Physiother Theory Pract. 2009;25:55-68.

9. Olney SJ, Nymark J, Brouwer B, et al. A randomized controlled trial of supervised versus unsupervised exercise programs for ambulatory stroke survivors. Stroke. 2006;37:476-481.

10. West T, Bernhardt J. Physical activity in hospitalised stroke patients. Stroke Res Treat. 2012;2012:813765.

11. Thieme H, Mehrholz J, Pohl M, et al. Mirror therapy for improving motor function after stroke. Cochrane Database Syst Rev. 2012;(3):CD008449.
12. Michielsen ME, Selles RW, van der Geest JN, et al. Motor recovery and cortical reorganization after mirror therapy in chronic stroke patients: a phase II randomized controlled trial. Neurorehabil Neural Repair. 2011;25:223-233.

13. Ada L, Dorsch S, Canning CG. Strengthening interventions increase strength and improve activity after stroke: a systematic review. Aust J Physiother. 2006;52:241-248.

14. Demeurrisse G, Demol O, Robaye E. Motor evaluation in vascular hemiplegia. Eur Neurol. 1980;19:382-389.

15. Sunderland A, Tinson D, Bradley L, et al. Arm function after stroke: an evaluation of grip strength as a measure of recovery and a prognostic indicator. $J$ Neurol Neurosurg Psychiatry. 1989;52:1267-1272.

16. Winward C, Halligan P, Wade D. The Rivermead Assessment of somatosensory performance: standardisation and reliability data. Clin Rehabil. 2002;16:523-533.

17. Bohannon R, Smith M. Inter-rater reliability of a Modified Ashworth Scale of muscle spasticity. Phys Ther. 1987;67: 206-207.

18. Wilson B, Cockburn J, Halligan P. Development of a behavioral test of visuo-spatial neglect. Arch Phys Med Rehabil. 1987;68:98-102.

19. Platz T, Pinkowski C, van Wijck F, et al. Reliability and validity of arm function assessment with standardized guidelines for the Fugl-Meyer Test, Action Research Arm Test and Box and Block Test: a multicentre study. Clin Rehabil. 2005;19:404-411.

20. Collen FM, Wade DT, Robb GF, et al. The Rivermead Mobility Index: a further development of the Rivermead Motor Assessment. Int Disabil Stud. 1991;13:50-54.

21. Tyson SF, DeSouza LH. Development of the Brunel Balance Assessment: a new measure of balance disability post stroke. Clin Rehabil. 2004;18:801-810.

22. Hennessey ML, Rumrill PD. Treatment fidelity in rehabilitation research. J Vocal Rehabil. 2003:19;123-126.

23. Thieme H, Bayn M, Wurg M, et al. Mirror therapy for patients with severe arm paresis after stroke. Clin Rehabil. 2012;27:314-324.

24. Dohle C, Püllen J, Nakaten A, et al. Mirror therapy promotes recovery from severe hemiparesis. Neurorehabil Neural Repair. 2009;23:209-217.

25. Deconinck FJ, Smorenburg AR, Benham A, Ledebt A, Feltham M, Savelsbergh GJ. Reflections on mirror therapy a systematic review of the effect of mirror visual feedback on the brain [published online August 26, 2014]. Neurorehabil Neural Repair. doi:10.1177/1545968314546134. 